\title{
ESB PlatForm INTEGRATING KNIME DATA MINING TOOL ORIENTED ON INDUSTRY 4.0 BASED ON ARTificial Neural Network Predictive MAINTENANCE
}

\author{
Alessandro Massaro, Vincenzo Maritati, Angelo Galiano, Vitangelo Birardi, \\ Leonardo Pellicani \\ Dyrecta Lab, IT research Laboratory, via Vescovo Simplicio,45, 70014 Conversano \\ (BA), Italy
}

\begin{abstract}
In this paper are discussed some results related to an industrial project oriented on the integration of data mining tools into Enterprise Service Bus (ESB) platform. WSO2 ESB has been implemented for data transaction and to interface a client web service connected to a KNIME workflow behaving as a flexible data mining engine. In order to validate the implementation two test have been performed: the first one is related to the data management of two relational database management system (RDBMS) merged into one database whose data have been processed by KNIME dashboard statistical tool thus proving the data transfer of the prototype system; the second one is related to a simulation of two sensor data belonging to two distinct production lines connected to the same ESB. Specifically in the second example has been developed a practical case by processing by a Multilayered Perceptron (MLP) neural networks the temperatures of two milk production lines and by providing information about predictive maintenance. The platform prototype system is suitable for data automatism and Internet of Thing (IoT) related to Industry 4.0, and it is suitable for innovative hybrid system embedding different hardware and software technologies integrated with ESB, data mining engine and client web-services.
\end{abstract}

\section{KEYWORDS}

ESB, Data Mining, KNIME, Industry 4.0, Predictive Maintenance, Artificial Neural Networks (ANN), MLP neural network.

\section{INTRODUCTION}

Open source Enterprise Service Bus (ESB) platforms have been discussed in the scientific research as important issues for IT architects working on Service Oriented Architecture (SOA) and for Enterprise Application Integration (EAI) [1]-[3]. Particular attention has been focused on the performance of WSO2 open source ESB able to manage simultaneously 50 threads [3] and to integrate different functionalities such as enterprise integration patterns, deliverable of all ESB features, completion of SOA, SOA governance, graphical ESB development, compostable architecture, cloud integration platform, a vailability of cloud connectors and of lagacy adapters, ultrafast performance (low computational cost), security and identify management, and open business model [4]. Concerning Industry 4.0, information digitization, and integration of different technologies are important elements for the development of connected and adaptive productions [5]. In this direction ESB could support Enterprise Integration Patterns (EIPs) about the use of enterprise application integration and message-oriented middleware in pattern language forms [1],[6]. In this direction data mining engines could improve the best pattern as for pattern-based manufacturing process optimization [7]. Interesting applications of data mining and artificial intelligence (A.I.) in industrial production process are in maintenance [8], 
in predictive maintenance reading Internet of Thing (IoT) sensor data [9],[10],[11], and generally in predictive analytics [12]. As open source data mining tools oriented on research we remember R language, RapidMiner, Weka, KNIME, Orange Canvas, and Tanagra [13],[14]. In particular KNIME is suited for web service connection [15], thus representing a good candidates for integration in an ESB network. Also Machine to Machine (M2M) systems could be integrated in a ESB network improving big data storage systems as Cassandra technology [16]. According with the requirements found in the state of the art, authors have developed in this work the information system infrastructure reported in Fig. 1 (a), which is related to an innovative integrated ESB system suitable for data transfer in Industry 4.0 processes. The layout has been designed by Aris Express, a graphical tool useful for process simulation [17]. In the proposed scheme are indicated the following modules:

- WSO2 Complex Event Processor (CEP): this module is suitable for the efficient management of processes and for the event scheduling; it helps to identify events and patterns from multiple data sources, analyze their impacts, working on them in real time;

- WSO2 Analytics: this module collects events through multiple transports and messaging formats; using streaming SQL to process streams, it detects complex events, performs prediction using machine learning models, and generates and notifies alerts in real-time by visualizing them with real time dashboards;

- WSO2 Machine Learner: the module of Machine Learner allows to process data by data mining algorithms using predefined parameters; for the best control of algorithms outputs, and for the choice of different algorithms typologies is required an external data mining tool; the algorithms that this module implements are related to numerical prediction (linear regression, ridge regression, lasso regression, random forest regression), binary classification (Logistic Regression SGD, Support Vector Machines SVM-), multiclass classification (logistic regression L-BFGS, Decision Tree, Random Forest Classification, Naive Bayes), clustering and anomaly detection (k-Means), anomaly detection, and deep learning (Stacked Autoencoders);

- WSO2 IoT Server: this module is useful for direct communication between sensors of different technologies and ESB network; it exposes an API to power a mobile app allowing users to monitor and control different devices or sensors;

- External data mining tool (KNIME): this external tool is able to improve advance analytics related to predictive maintenance of the production lines; the external tool is necessary to control data processing, to set the best parameters and to choice new machine learning algorithms different to those implemented in WSO2 Machine Learner modules, such as Artificial Neural Networks (ANN) [18],[19] well suitable for predictive maintenance.

Starting from the infrastructure of Fig. 1 (a) authors focused the attention on the application of the external data mining tool working by means of a web client service interfaced with the prototype ESB network (see dashed part sketched in Fig. 1 (b)). In this way authors will apply the most important functionalities of the prototype infrastructure enhancing the innovative aspects of the research. The paper is structured as follows:

- Development of the prototype of Fig. 1 (b) by performing a basic first test related to the check of data transfer between client web service, KNIME and ESB; the outputs of this test will prove the correct functionality of a KNIME workflow managed by ESB; this preliminary test is fundamental in order to understand data flow process in the prototype system; 
International Journal of Artificial Intelligence and Applications (IJAIA), Vol.9, No.3, May 2018

- Development of the prototype of Fig. 1 (b) by improving an advanced algorithm based on ANN and simulating predictive maintenance of two production lines controlled by temperature sensors.

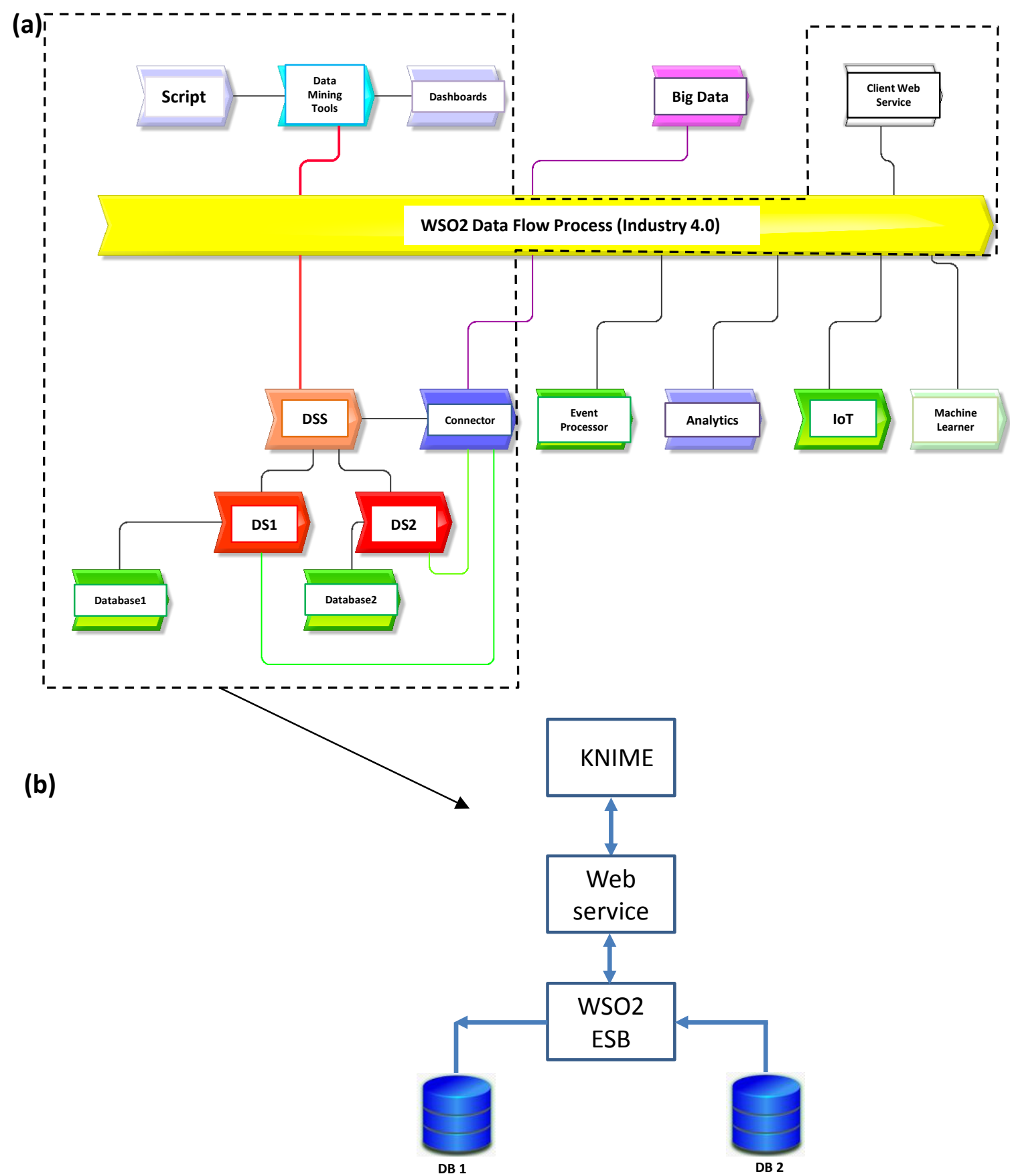

Figure 1. (a) ESB prototype adopted in the industrial project. (b) Part of the developed prototype concerning interconnection with KNIME tool. 


\section{First And Second Test Of The Prototype Platform}

Figure 1 (b) illustrates the prototype part tested for checking data transfer and applied for the implementation of artificial neural network algorithms. Specifically in Fig. 1 (b), ESB WSO2 platform manages two different relational database management system (RDBMS), named DB 1 and DB 2 which are datasources processed by a KNIME workflow, through a web-service. In the next paragraphs will be discussed the test performed by applying the network prototype.

\subsection{First test checking data flow in ESB infrastructure and main functionalities}

The first implementation of the data flow of Fig. 1 (b) is related to a preliminary check useful to verify ESB data management. Specifically two databases have been created by MySQL tool. The first RDBMS has been structured in localhost by the following SQL script:

CREATE DATABASE PIMA;

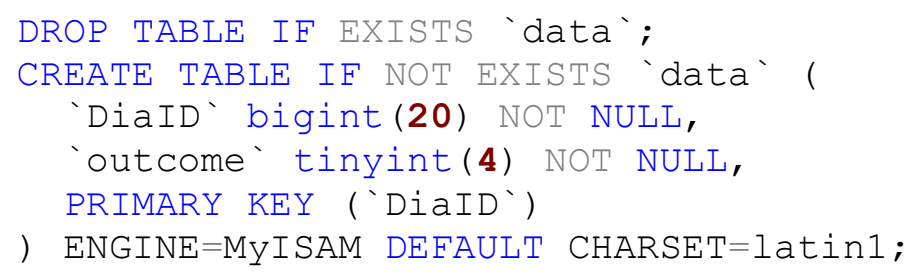

A second RDBMS has been created on other machine attainable by an IP address. The script used for creation of the second RDBMS is the following one (768 patient data in medical application):

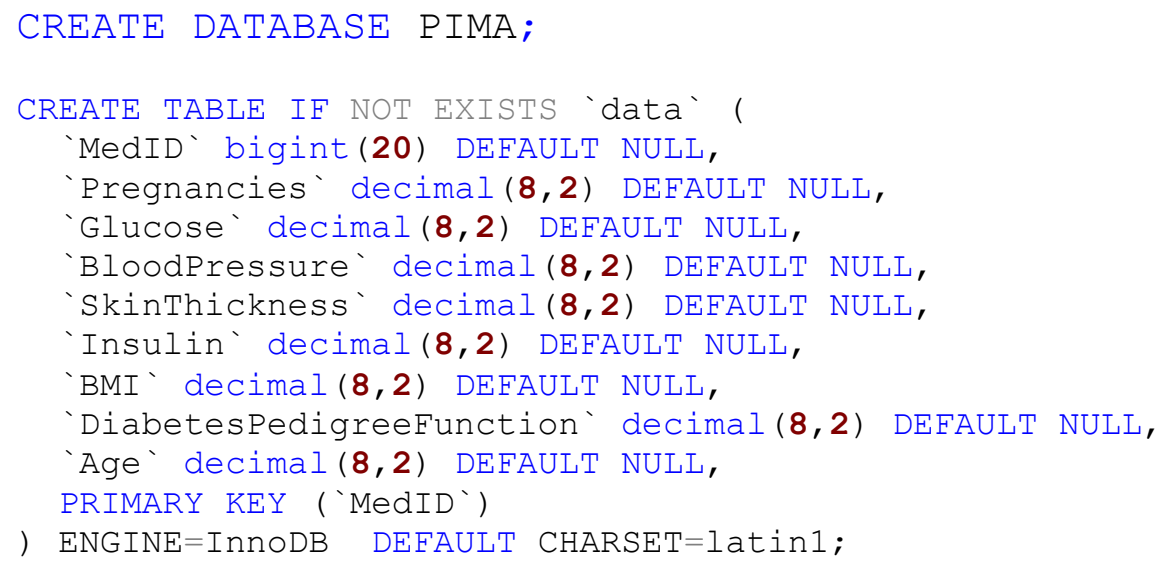

For the database population has been adopted Python environment by using the open source editor Jupyter Notebook, that is a script editor that allows to execute script parts independently from the others and that allows also to insert documentation in the product code. Successively has been created a data service on WSO2 DSS module able to connect to two different RDBMS, to aggregate data (in this test is performed a merge process between personal data of the first database with medical measured data of the second one), and to provide processed data at ESB output. The WSO2 DSS platform has been installed locally, as one of the two MySQL servers. In the WSO2 panel windows have been created the two datasources (named in Fig. 1 (a) by DS 1 and DS 2) by means the command "Add Datasource". In Fig. 2 is reported the screenshot related the final creation of both datasources. In order to check the datasource management of WSO2 DSS 
ESB has been executed the following query SQL script (following the step "Next"-“Add new query"):

Select MEDID, Pregnancies, Glucose, BloodPressure,Skinthickness, Insulin, BMI, DiabetesPedigreeFunction, Age from data where MEDID = :DIAID

The response generation of the SQL script is illustrated in Fig. 3 thus representing the correct datasource management of the ESB.

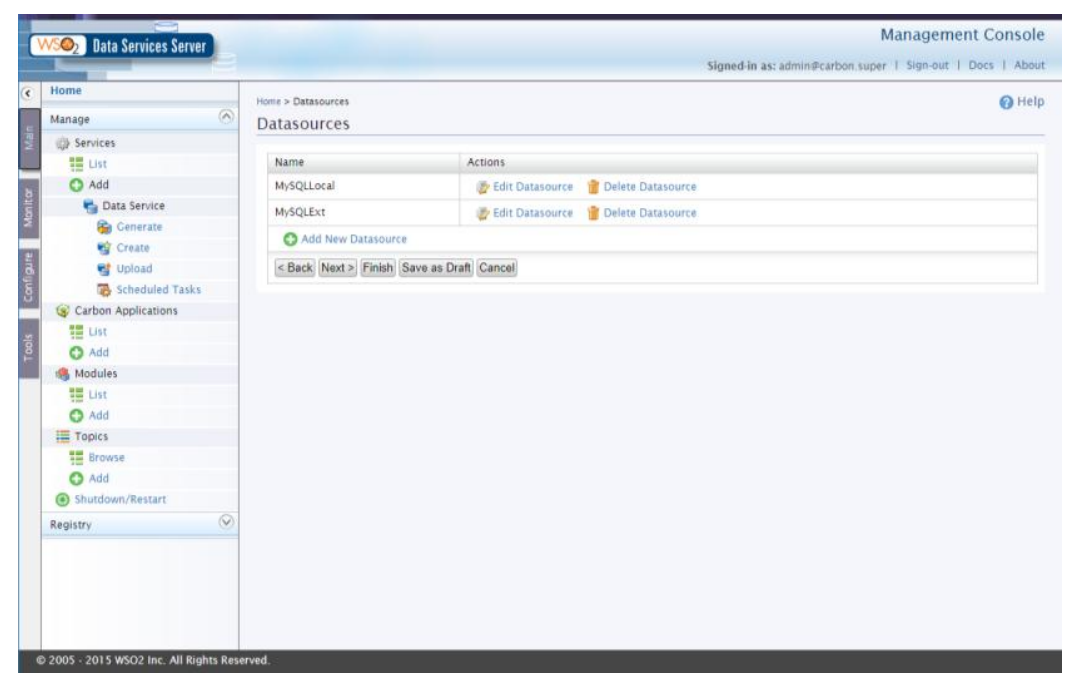

Figure 2. Screenshot of correct creation of the two datasources.

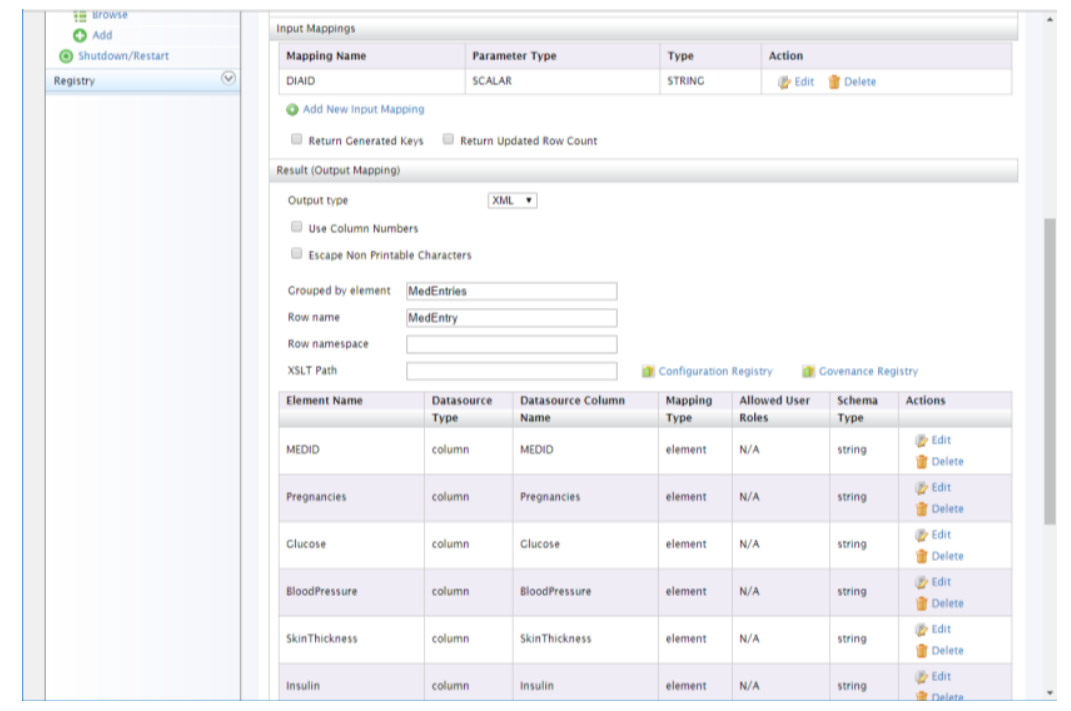

Figure 3. Screenshot of test.

The second datasource has been checked by the following script:

\section{Select id as diad, outcome from data limit 5}

After the check of datasource management in ESB has been tested the data service by creating a Python script in a KNIME object. Zeep library has been adopted for the client web-service and Pandas library has been used for KNIME interaction. The script used for the libraries call is the following: 
from zeep import Client

from zeep.wsse.username import UsernameToken

import pandas as pd

import zeep

Below is indicated the script able to activate the client web-service (WSDL1.0 and WSDL2.0):

$$
\begin{aligned}
& \text { user = 'admin' } \\
& \text { password = 'admin' } \\
& \text { wsdl_url = 'http://192.168.0.102:9763/services/Pimalndians?wsdl' } \\
& \text { client = Client(wsdl_url, wsse=UsernameToken(user, password)) }
\end{aligned}
$$

After the webservice call, the data has been converted in an ordered data list and successively in a dataframe by the following script

$d f=p d$.DataFrame $\left(m y \_d a t a\right)$

df.columns = [ 'id', 'outcome', 'Pregnancies', 'Glucose', 'BloodPressure', 'SkinThickness', 'Insulin', 'BMI', 'DiabetesPedigreeFunction', 'Age']

print (df)

thus providing the following output (are shown the first 5 rows )

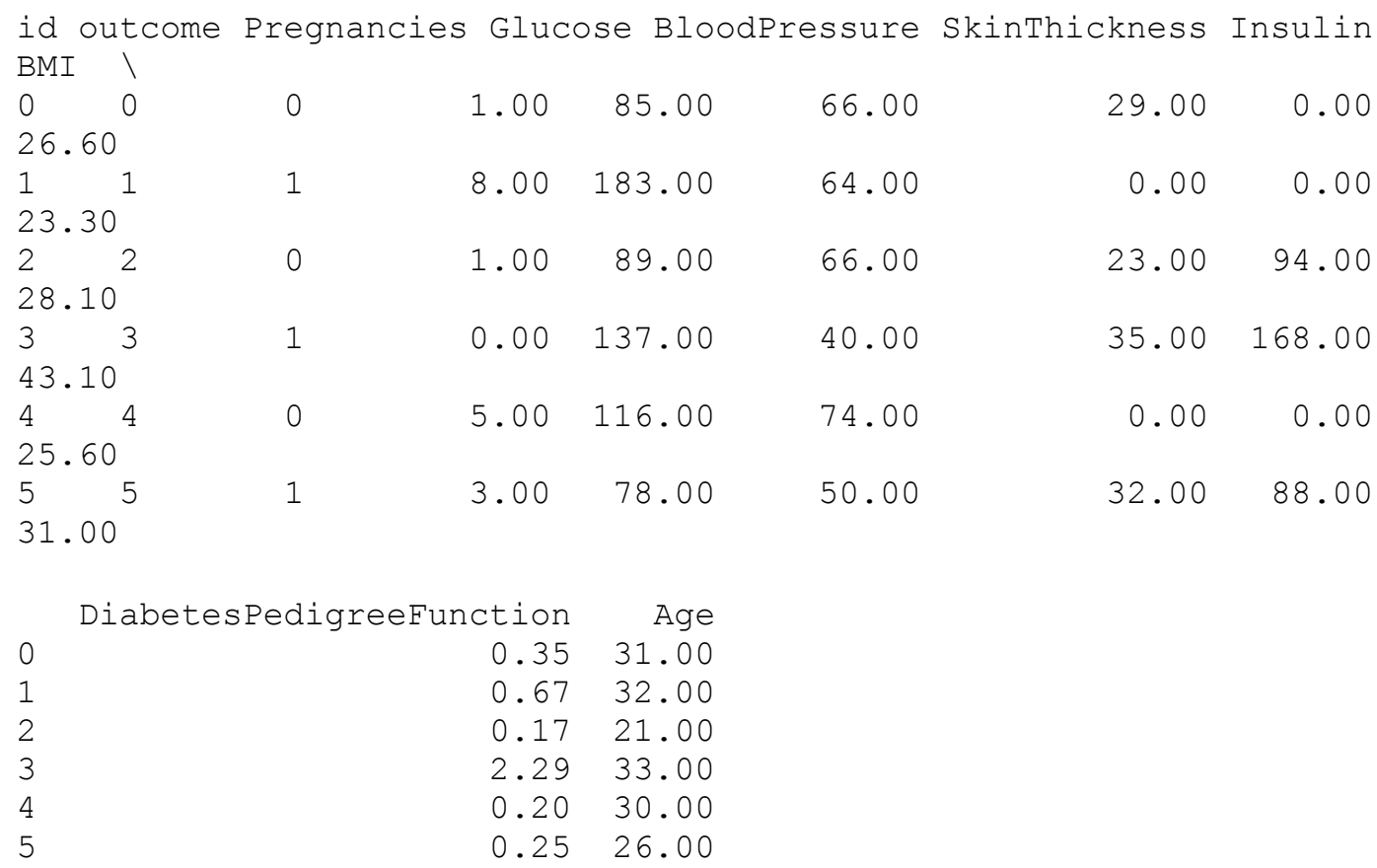

The created dataframe will be passed to KNIME node able to process data.

In Fig. 4 is shown the KNIME workflow designed for the first test, where in the first node named "Python Source" has been executed the following python script:

from zeep import Client

from zeep.wsse.username import UsernameToken

import pandas as $p d$

import zeep 

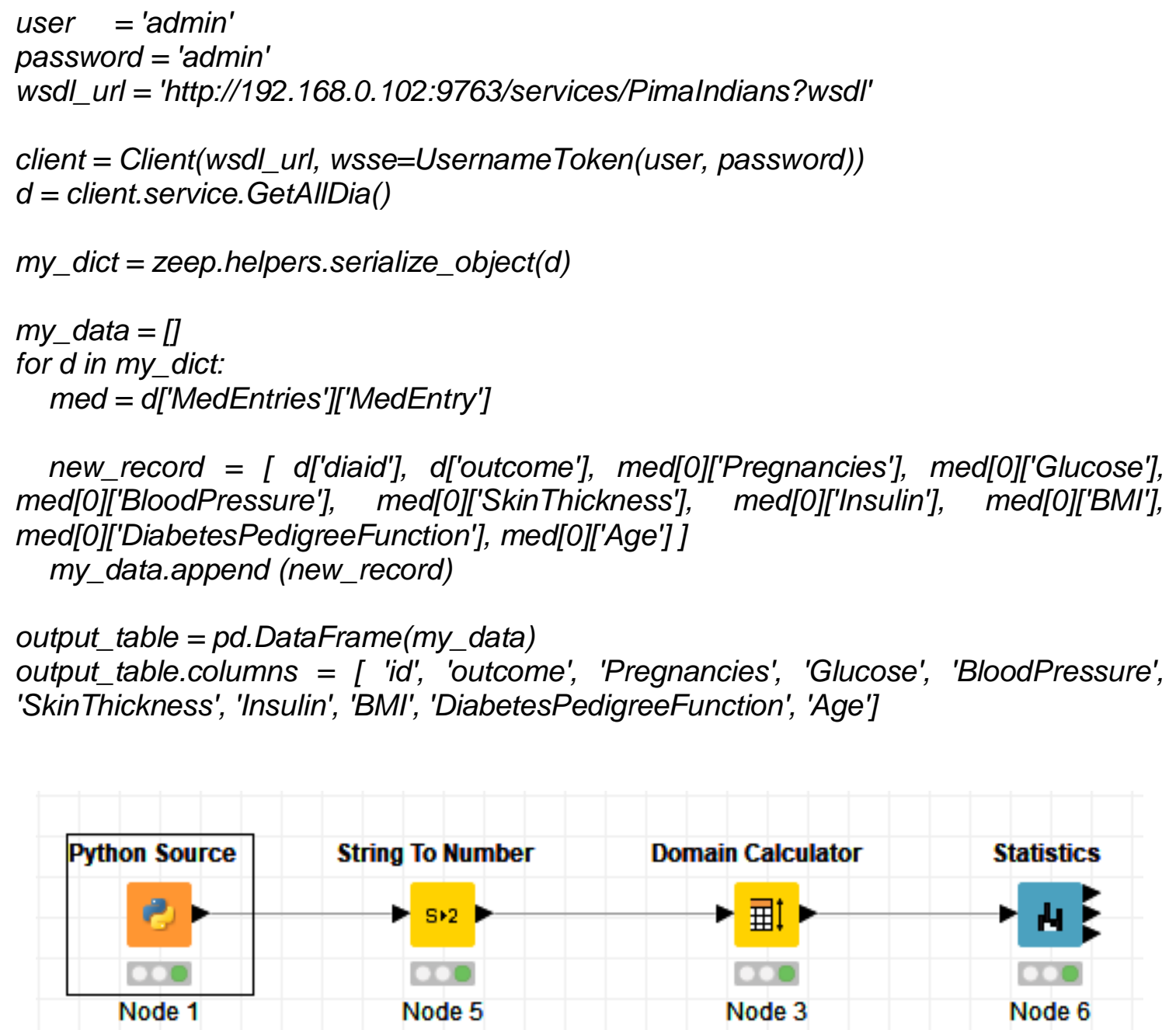

Figure 4. KNIME workflow of the first test.

The node "String to Number" will convert the string in data number to process, and the node "Domain Calculator" will select the attributes to process (in this case all the attributes are considered). In Fig. 5 is illustrted the output of the "Statistic" node proving the ESB-WSO2KNIME data tranfer and data processing. 


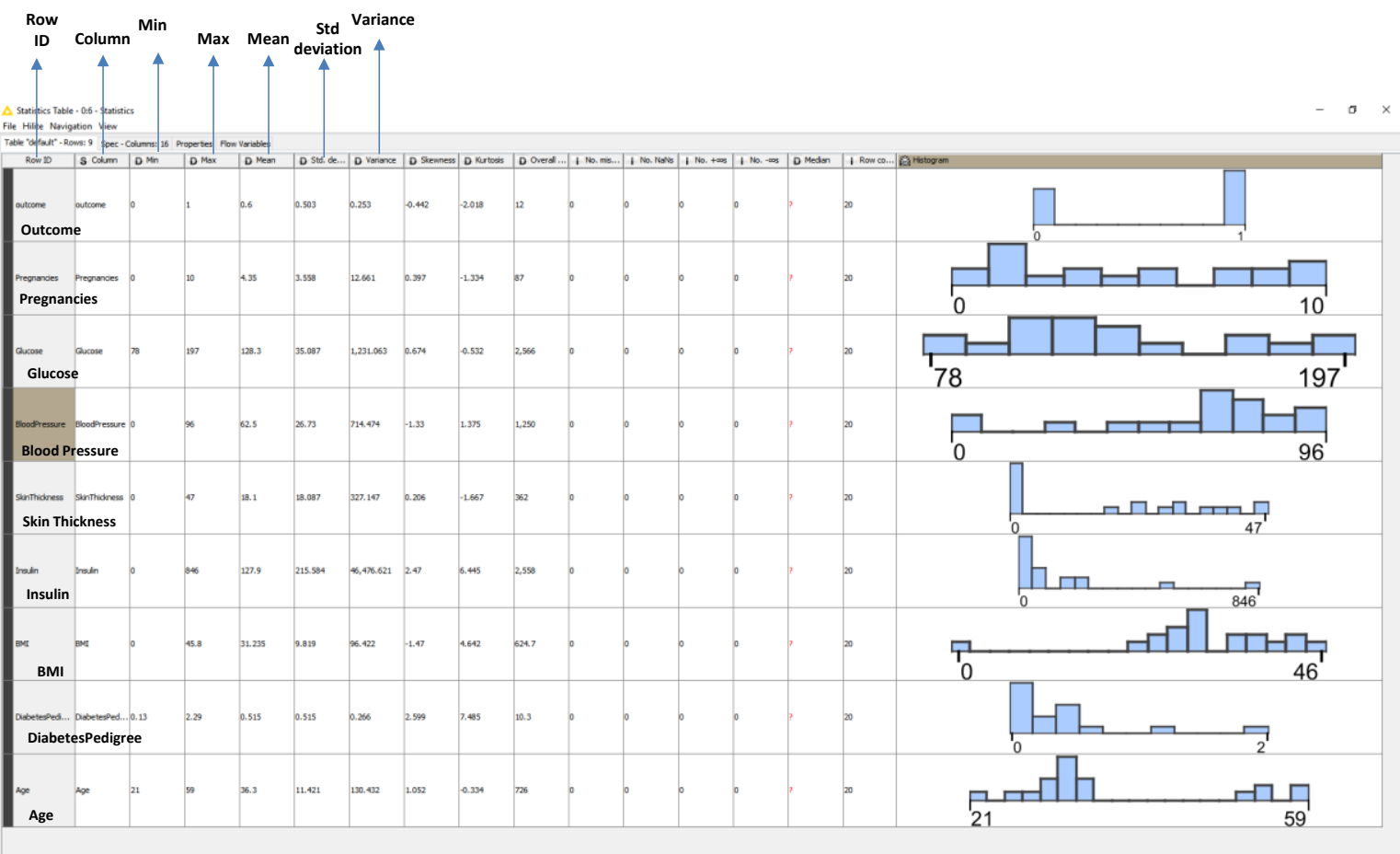

Figure 5. KNIME: output of the "Statistic Node" (Node 6).

\subsection{Second Test oriented on Industry 4.0 application: application of KNIME neural networks for predictive maintenance of two production lines}

The first example is useful to understand the basic principle of data management of the prototype system. In this section, after an introduction of ANN, will be implemented the test of predictive maintenance tool.

\subsubsection{MLP Neural Network Theory}

An MLP (or Artificial Neural Network - ANN) with a single hidden layer can be represented graphically as in Fig. 6

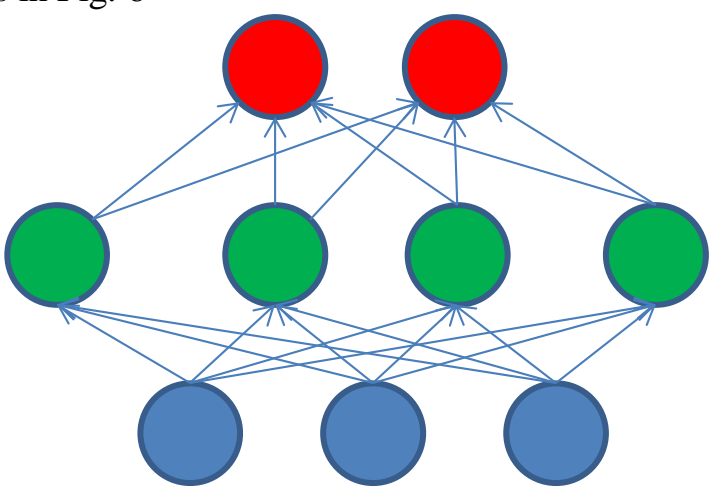

Output layer

Hidden layer

Input layer

Figure 6. MLP neural network. 
An MLP consists of multiple layers of nodes in a directed graph, with each layer fully connected to the next one. Except for the input nodes, each node is a neuron (or processing element) with a nonlinear activation function.

Formally a one-hidden-layer MLP is a function $f: R^{D} \rightarrow R^{L}$ being $D$ the size of the input vector $x$ and $L$ the size of the output vector $f(x)$ defined as:

$$
f(x)=G\left(v^{(2)}+W^{(2)}\left(s\left(v^{(1)}+W^{(1)} x\right)\right)\right)
$$

where $v^{(1)}$ and $v^{(2)}$ are the bias vectors, $W^{(1)}$ and $W^{(2)}$ the weight matrices, and $G$ and $s$ the activation functions. The following vector

$$
h(x)=\varphi(x)=s\left(v^{(1)}+W^{(1)} x\right)
$$

will define the hidden layer, and $W^{(1)} \in R^{D \times D h}$ is the weight matrix connecting the input vector to the hidden layer. $W_{i}^{(1)}$ will represent the weights from the input units to the $i$-th hidden unit. Typically the activation function $s$ assumes the following forms

$$
\begin{aligned}
& \tanh (a)=\left(e^{a}-e^{-a}\right) /\left(e^{a}+e^{-a}\right) \\
& \operatorname{sigmoid}(a)=1 /\left(1+e^{-a}\right)
\end{aligned}
$$

The output vector is defined by:

$$
o(x)=G\left(v^{(2)}+W^{(2)} h(x)\right)
$$

To train an MLP the model will be learned, the set of parameters to learn is

$$
\gamma=\left\{W^{(2)}, v^{(2)}, W^{(1)}, v^{(1)}\right\}
$$

thus obtaining the gradients

$$
\partial l / \partial \gamma
$$

of the back propagation algorithm adopted for the model training. Back propagation algorithm is a supervised learning method which can be divided into two phases: propagation and weight update. The two phases are repeated until the performance of the network is good enough. In back propagation algorithms, the output values are compared with the correct answer to compute the value of some predefined error-function. By various techniques, the error is then fed back through the network. Using this information, the algorithm adjusts the weights of each connection in order to reduce the value of the error function by some small amount. After repeating this process for a sufficiently large number of training cycles, the network will usually converge to some state where the error of the calculations is small. In this case, one would say that the network has learned a certain target function.

\subsubsection{Second test oriented on predictive maintenance}

Temperature measurement is one of approach to control the production and some industrial machines. For this purpose authors analyse in this section two parts of two milk production 
lines related the pasteurization process. In this process a key parameter to monitor is the temperature: a good pasteurization process works within a range of $70{ }^{\circ} \mathrm{C} \div 75{ }^{\circ} \mathrm{C}$, for this reason it is important to control temperature trend during the time. In Fig. 7 (a) is illustrated the simplified prototype infrastructure adapted to the predictive maintenance of milk pasteurization process of two distinct production lines placed in different locations. The main function of the ESB is the simultaneous control of pasteurization process in both different lines by predicting possible anomalies and alerting conditions. Some researchers suggested the idea to create a reference model based on the use of the use of Multilayered Perceptron-MLP- neural networks and on temperature information useful to classify thermal defects in predictive maintenance [20]. In this direction authors applied the same MLP algorithm of KNIME tool by considering the client web-service related to the prototype of Fig. 1 (b), where the first datasource is defined by the temperature dataset of the attribute $\mathrm{T} 1$ (temperature of the production line 1), and the second datasource is defined by the temperature dataset of the attribute T2 (temperature of the production line 2). The MLP algorithm can be viewed as a logistic regression classifier where the input is first transformed using a learnt non-linear transformation. This transformation projects the input data into a space where it becomes linearly separable by applying the theory discussed in the section 2.1.1.. with a single hidden layer as intermediate layer sufficient to make MLPs as an universal approximator.

For the training of the MLP neural network model (learner of the model) has been applied the efficient RProp algorithm for multilayer feed-forward networks defined in [21],[22]: RProp performs a local adaptation of the weight-updates according to the behaviour of the error function. In this test case the neural network parameters are set as follows: the maximum number of iteration equals to 100 , the number of hidden layers is equals to 1 , the number of hidden neurons per layer is equals to 10 , the class to predict is Coll (temperatures of each lines).

The predictive maintenance will be performed by comparing the predicted values with real temperature trends of both datasources: in the case of a convergence will be a correct functionalities of the line production machines, otherwise will happen potential anomalies. 
(a)

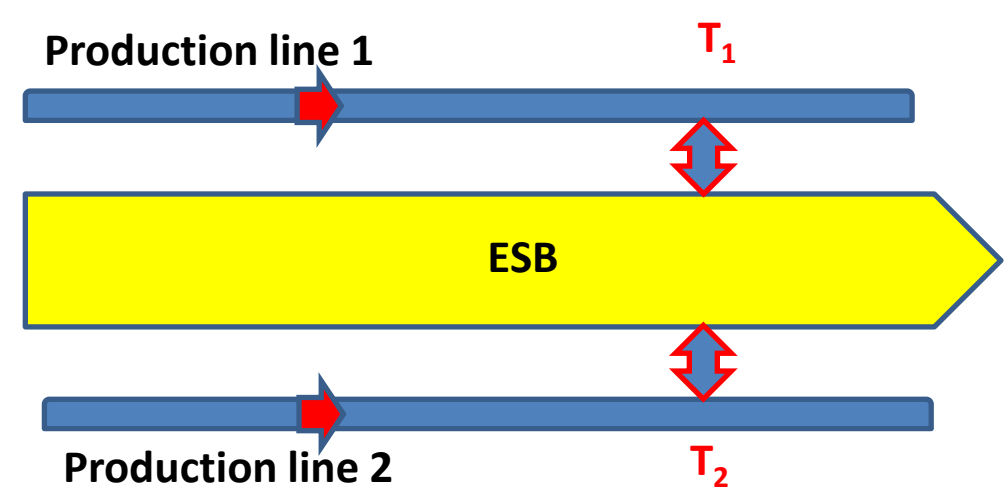

(b)

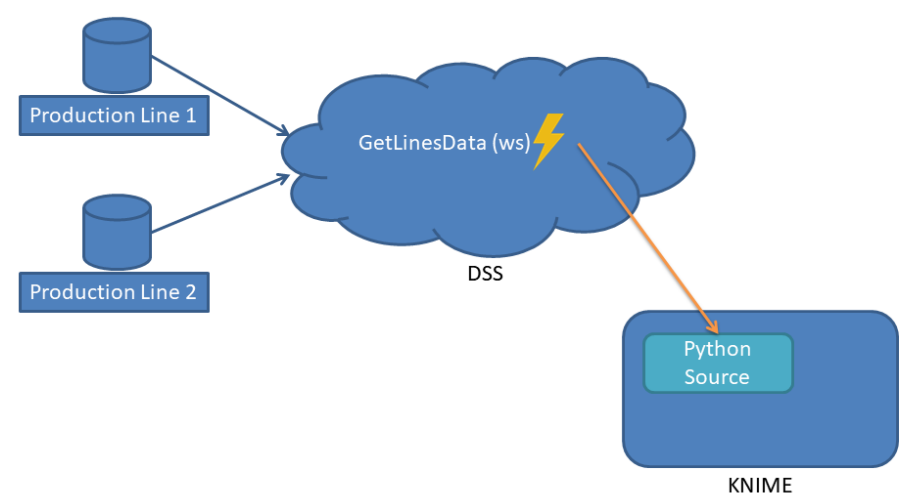

Figure 7. (a) Simplified model related to predictive maintenance process concerning the working simulation of milk pasteurization machines.(b) Architecture integrating the simplified model.

In Fig. 7 (b) is illustrated the architecture integrating web-service in the prototype ESB infrastructure interfaced with KNIME MLP neural network algorithm.

Below is the Python code used to query the web-service "GetLinesData", instanced on the WSO2 DSS and related to the construction of a dataset based on the data retrieved from the two MySQL databases where are stored the data collected on two different production lines:

from requests import Session

from requests.auth import HTTPBasicAuth

from zeep import Client

from zeep.transports import Transport

from zeep.wsse.username import UsernameToken

from pandas.io.json import json_normalize

import pandas as pd

import zeep

user = 'admin'

password = 'admin'

wsdl_url = 'http://192.168.0.102:9763/services/GetLinesData?wsdl'

client = Client(wsdl_url, wsse=UsernameToken(user, password $)$ )

lines_data $=$ client.service.GetLinesData()

$m y \_d i c t=z e e p . h e l p e r s . s e r i a l i z e \_o b j e c t(d)$ 
my_data $=[]$

for d in my_dict:

new_record $=$ [lines_data ['id'], lines_data ['Line1'], lines_data ['Line2'], lines_data ['Timestamp'] ]

my_data.append (new_record)

output_table $=$ pd.DataFrame $\left(m y \_d a t a\right)$

output_table.columns $=$ [ 'id', 'Line1', 'Line2', 'Timestamp' $]$

In Fig. 8 is illustrated the KNIME workflow enabling the simultaneous pasteurization control executing the RProp Learner and the MLP Predictor. The input datasets of the proposed model are made by 168 values corresponding to the temperature measurements of seven days ( 1 value of each hour) of both production lines. The "Normalizer" object is necessary to process data in normalized temperature of a range between 0 and 1 . The "Partitioning" block is able to split 80 data for the learner module (first partition) and 88 data for the MLP predictor (second partition).

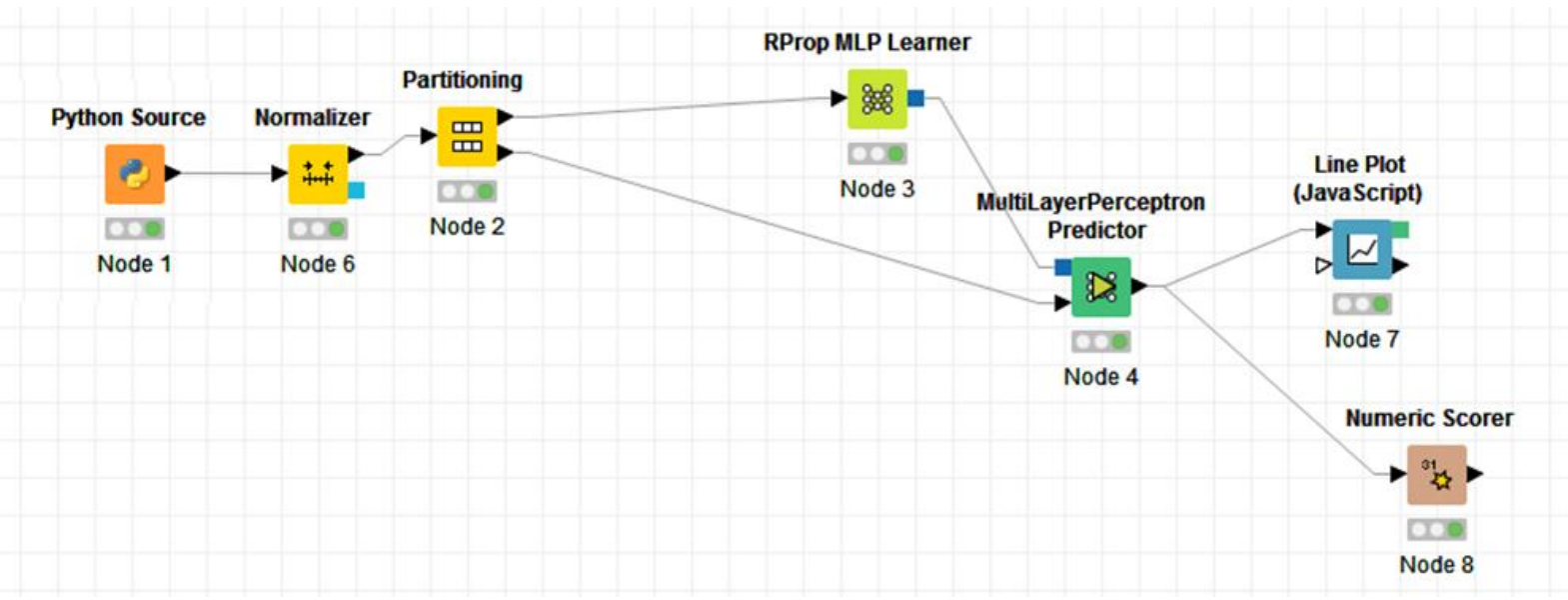

Figure 8. KNIME: MLP workflow model of predictive maintenance applied for each production line.

Figure 9 proves that Python code listed before has been loaded correctly in the KNIME engine. 
International Journal of Artificial Intelligence and Applications (IJAIA), Vol.9, No.3, May 2018

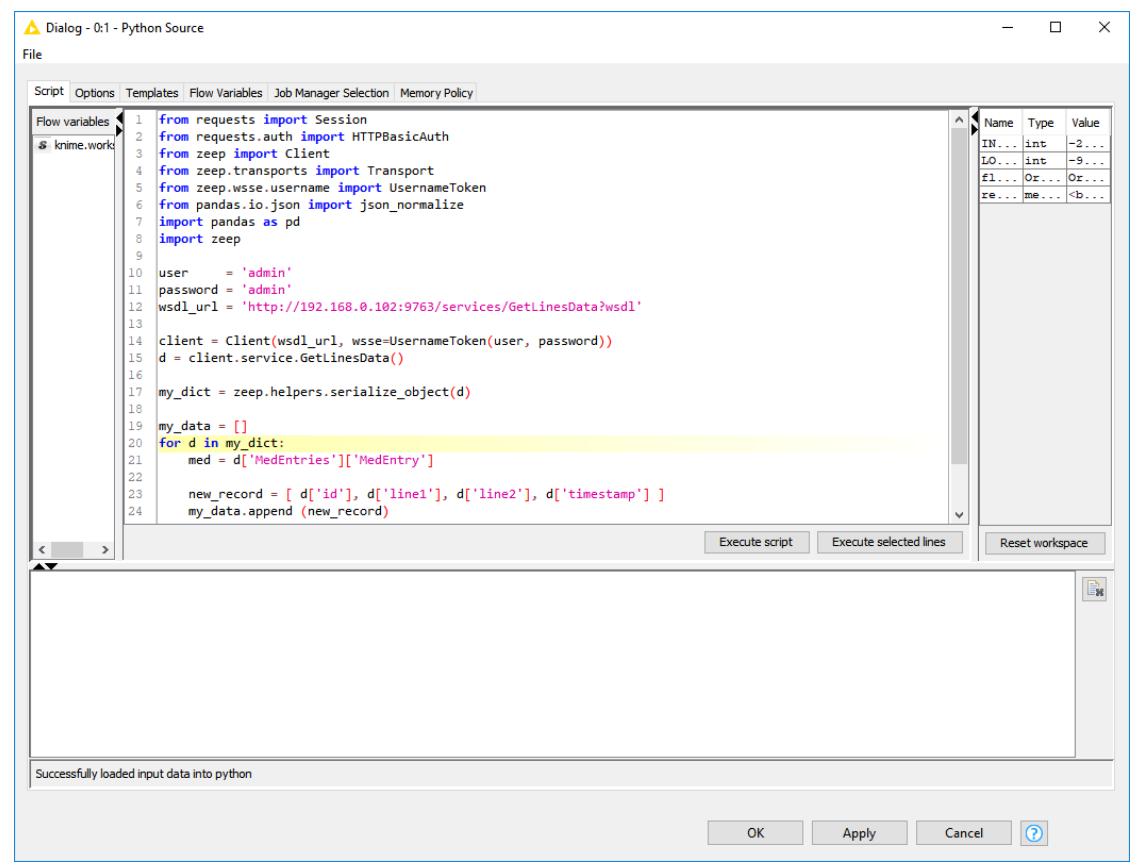

Figure 9. KNIME: configuration panel of the "Python Source" node 1.

As example In Fig. 10 are illustrated the first ten values of the "Partitionig" of the first production line, where the attribute Col0 indicates the hour attribute, and Coll the normalized measured temperature attribute. It is important to note that both partitions are characterized by different datasets.

\begin{tabular}{||l|l|l|}
\multicolumn{1}{|c|}{ Row ID } & \multicolumn{1}{c|}{ I Col0 } & D Col1 \\
\hline Row1 & 2 & 0.25 \\
\hline Row5 & 6 & 0.85 \\
\hline Row9 & 10 & 0.85 \\
\hline Row 10 & 11 & 0.85 \\
\hline Row13 & 14 & 0.85 \\
\hline Row14 & 15 & 0.85 \\
\hline Row15 & 16 & 0.85 \\
\hline Row16 & 17 & 0.85 \\
\hline Row18 & 19 & 0.85 \\
\hline Row19 & 20 & 0.75 \\
\hline
\end{tabular}

\begin{tabular}{|l|l|l|}
\hline \multicolumn{1}{|c|}{ Row ID } & \multicolumn{1}{c|}{ I Col0 } & D Col1 \\
\hline Row0 & 1 & 0 \\
\hline Row2 & 3 & 0.5 \\
\hline Row3 & 4 & 0.75 \\
\hline Row4 & 5 & 1 \\
\hline Row6 & 7 & 0.9 \\
\hline Row7 & 8 & 0.5 \\
\hline Row8 & 9 & 0.85 \\
\hline Row11 & 12 & 0.85 \\
\hline Row12 & 13 & 0.85 \\
\hline Row17 & 18 & 0.85 \\
\hline
\end{tabular}

Figure 10. KNIME "Partinioning" object: first and second partition of production line 1 (first 10 values).

In order to check the alarm condition has been defined a gap (see Fig. 11) of amplitude of 0.2 defined as an error bar of \pm 0.1 centered to the average amplitude of the prediction line. If real temperature values will overcome the threshold lines a potential anomaly will occur during the pasteurization process. If real temperature trend is within the gap region the system will work correctly as shown in the theoretical case of Fig.11. 


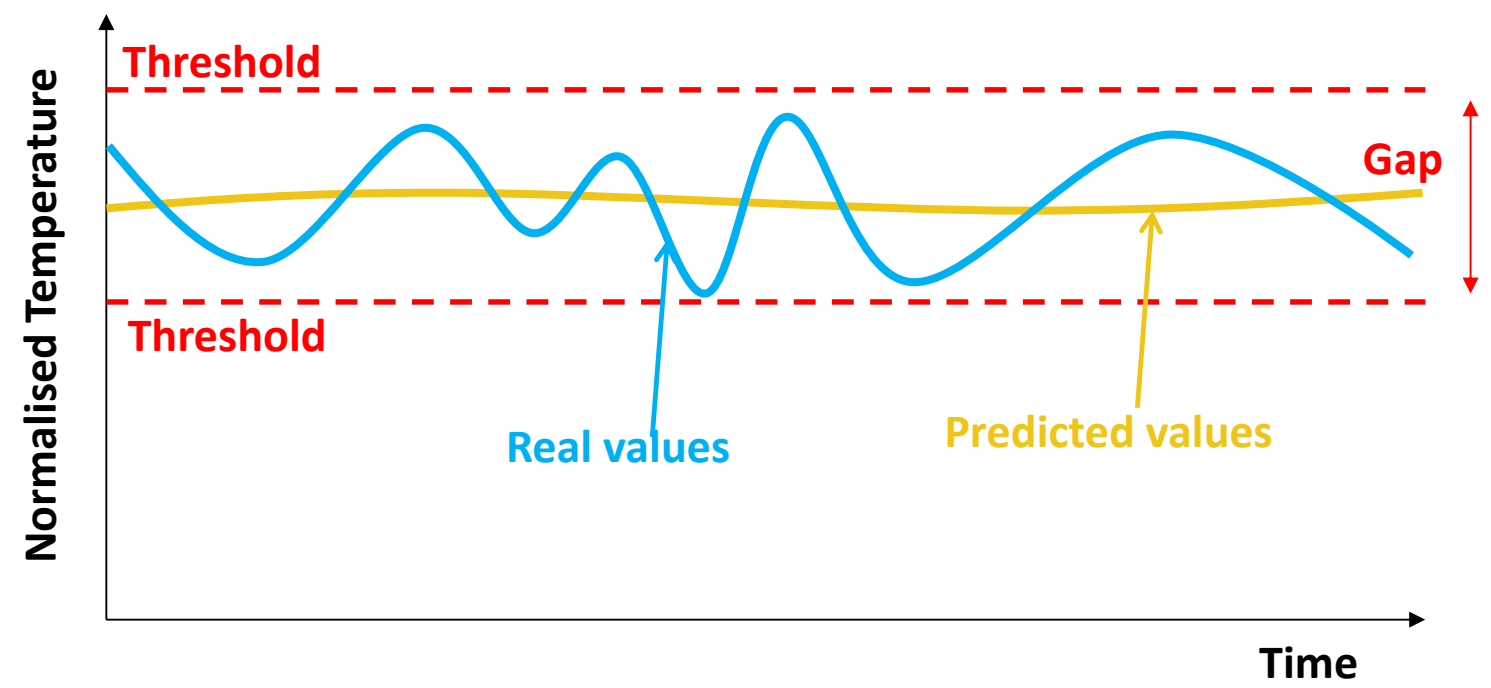

Figure 11. Theoretical good matching between predicted and real temperature values.

According with performed test on both the production lines is obtained the MLP neural network outputs of Fig. 12 and Fig. 13, where are compared the predicted temperatures versus the real ones. Figure 12 exhibits only one alert condition: after the first transitory related to a switching on condition, temperature values are mainly within the gap region, by indicating that the production line works correctly. In any case it is important to check the model in the next seven day in order to control other alert conditions: a recurrence of alert conditions will represent a potential risk of anomaly. In Fig. 13 is illustrated the test performed on the second production line. In this second case more alert conditions are checked thus indicating that the line could work badly.

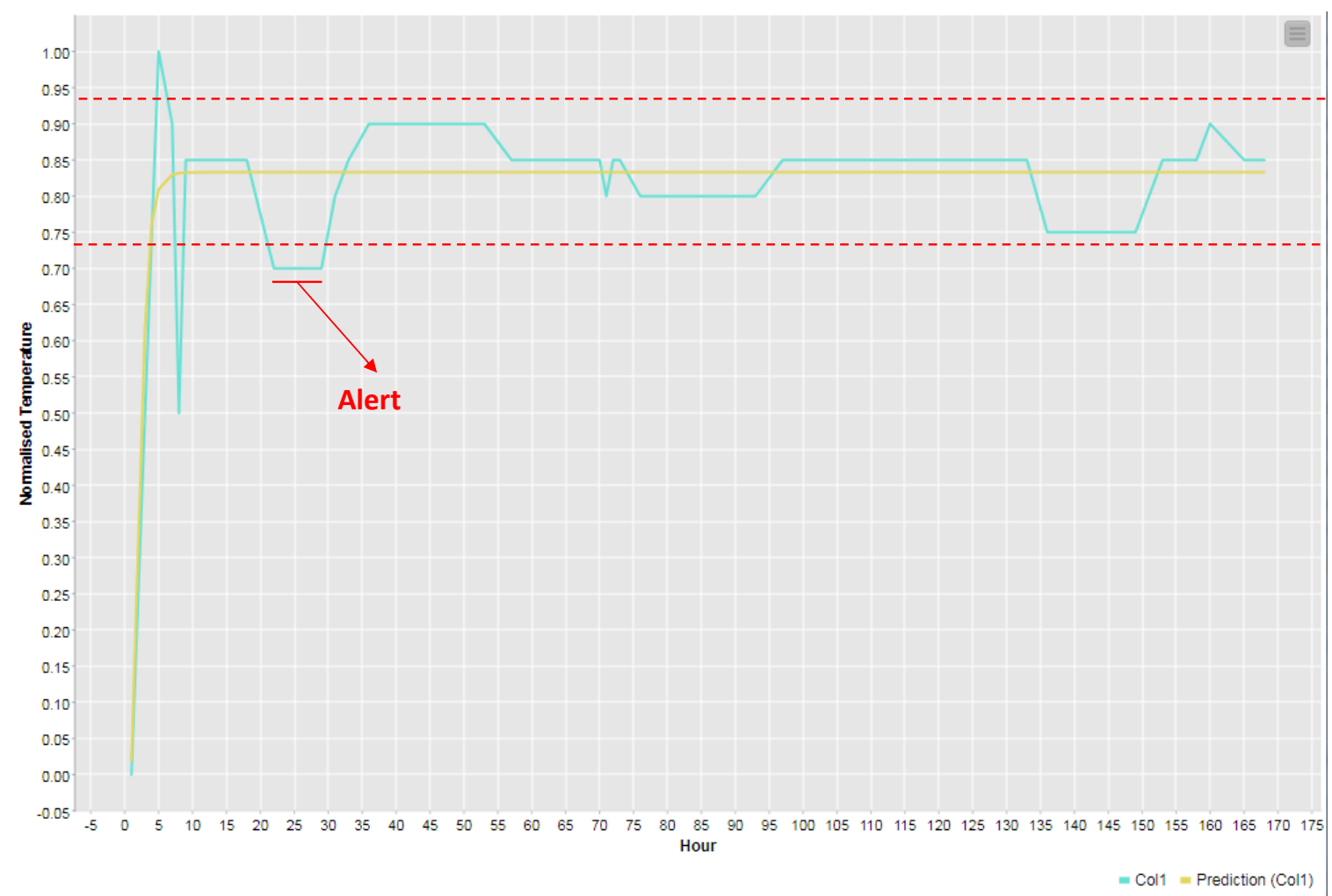

Figure 12. KNIME MLP model output: comparison between measured data and predicted ones of the production line 1. 


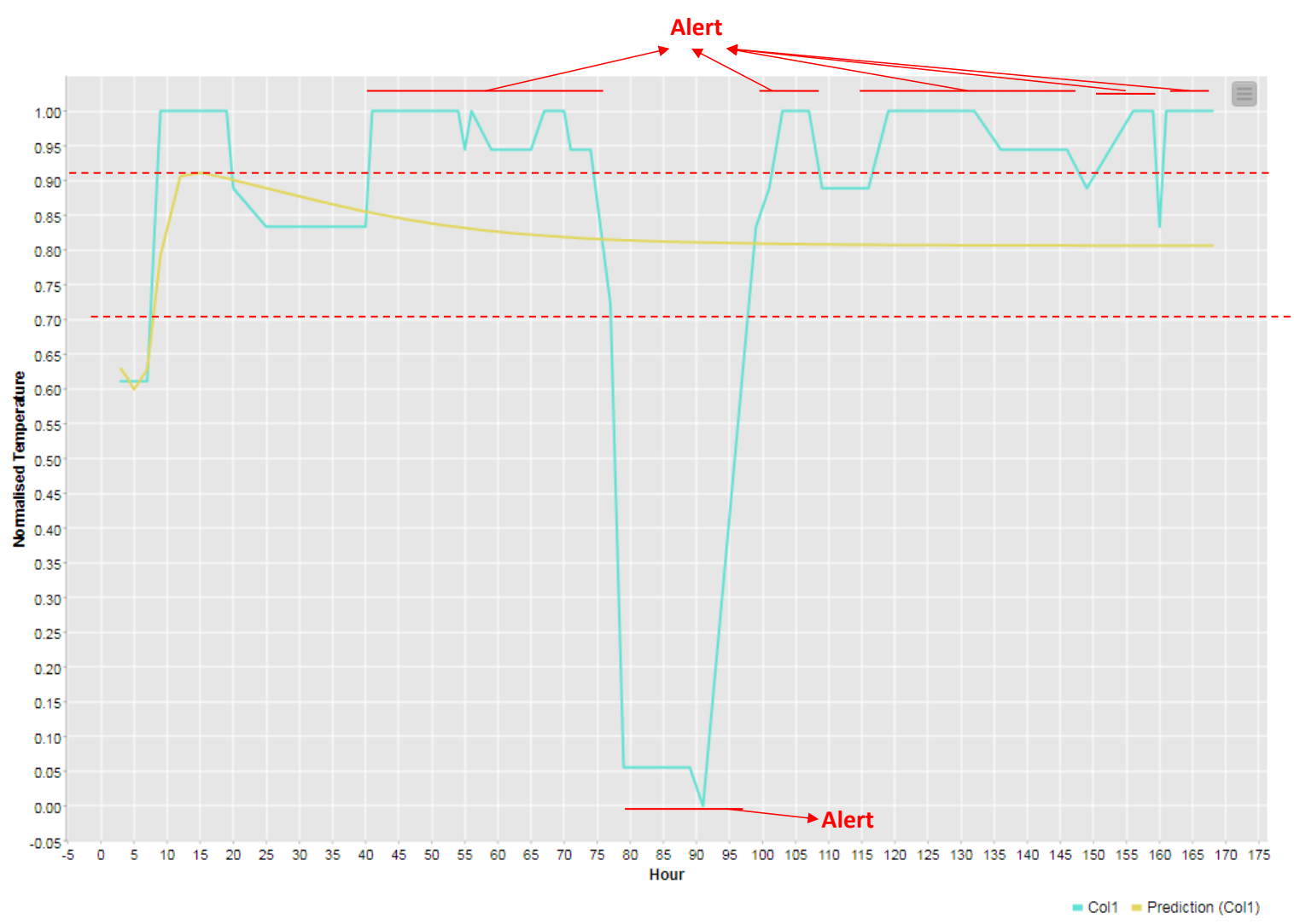

Figure 13. KNIME MLP model output: comparison between measured data and predicted ones of the production line 2 .

According with scoring values it is possible to confirm that the applied model is reliable (a mean absolute error equals to 0.04 is estimated). The proposed procedure can be adopted in different applications involving healthcare [23] and for mapping industrial processes [24]. The ANN could be implemented by means of a tailored architecture [25].

\section{CONCLUSION}

The goal of the proposed paper is to propose a data infrastructure model applicable in Industry 4.0 and suitable for control rooms. The performed tests prove that WSO2 ESB can be adopted to transfer data coming from different databases and processed by the external KNIME data mining engine through client-web services. The first example has been applied on medical data thus confirming that the prototype model is oriented on different fields including healthcare applications as for prevention of heart problem. Concerning industrial applications the ESB can connect different information systems related to different production line. As proved in work, predictive maintenance can provide important information about risks and production anomalies, and could be applied with historical measured data in order to predict product quality by means of provisional Xbar-charts and the R-charts, charts useful for the standard ISO 9001:2015. The development of ANN requires a proper architecture network. In this direction ESB infrastructure represent a good tool compatible with different operating systems and different hardware machines thus providing in the same time web-service facilities. The shown results proved the implementation feasibility of ANN in a ESB connected to a web-service system. The practical test of monitoring predictive maintenance of two milk production lines by MLP ANN directs the 
research in innovative solutions for information technologies oriented on industrial applications and industrial research.

\section{ACKNOWLEDGEMENTS}

The work has been developed in the frameworks of the Italian projects: "Sistemi software interfacciabili e modulari orientati alla comunicazione di Industria 4.0 e all' analisi avanzata dei dati: -ModularIndustry4.0-. [Interfacable and modular software systems oriented on Industry 4.0 communication and on advanced data analysis: -ModularIndustry4.0-]". The authors would like to thank the following researchers and collaborators: D. Barbuzzi, G. Birardi, B. Boussahel, V. Calati, D. Carella, A. Colonna, R. Cosmo, V. Custodero, L. D'Alessandro, G. Fanelli, M. Le Grottaglie, A. Leogrande, A. Lombardi, G. Lonigro, A. Lorusso, L. Maffei, S. Maggio, N. Malfettone, S. F. Massari, G. Meuli, R. Porfido, O. Rizzo, D. D. Romagno, N. Savino, P. Scagliusi, S. Selicato, G. Sicolo, M. Solazzo, M. M. Sorbo, D. Suma, F. Tarulli, E. Valenzano, V. Vitti and M. Dal Checco.

\section{REFERENCES}

[1] Hohpe, G., \& Woolf, B. (2004) "Enterprise Integration Patterns Designing, Building, and Deploying Messaging Solutions”, Addison-Wesley.

[2] Polgar, J. (2009) “Open Source ESB in Action”,IGI Publishing.

[3] Górski, T., \& Pietrasik, K. (2016) "Performance analysis of Enterprise Service Buses", Journal of Theoretical and Applied Computer Science, Vol. 10, No. 2, pp 16-32.

[4] Yenlo (2016) "ESB Comparison How to choose a reliable and fast ESB that fits your business needs", white paper.

[5] Fraunhofer Institute report: INDUSTRY 4.0 - CONNECTED, ADAPTIVE PRODUCTION. white paper available on line.

https://www.ipt.fraunhofer.de/content/dam/ipt/en/documents/broschures/Industry\%2040-

Connected\%20Adaptive\%20Production.pdf

[6] Theorin, A., Bengtsson, K., Provost, J., Lieder, M., Johnsson, C., Lundholm, T., Lennartson, B. (2017) "An Event-Driven Manufacturing Information System Architecture for Industry 4.0", International Journal of Production Research, Vol. 55, No.5, pp1297-1311.

[7] Gröger, C., Niedermann, F., Mitschang, B. (2012) "Data Mining-Driven Manufacturing Process", Proceedings of the World Congress on Engineering (WCE 2012), volume III, London, U.K..

[8] Bastos, P., Lopes, I., Pire, L. (2014) "Application of Data Mining in a Maintenance System for Failure Prediction. Safety, Reliability and Risk Analysis: Beyond the Horizon", Steenbergen et al. (Eds), Taylor \& Francis, pp933-940.

[9] Massaro, A., Galiano, A., Meuli, G., Massari, S. F. (2018) "Overview and Application of Enabling Technologies Oriented on Energy Routing Monitoring, on Network Installation and on Predictive Maintenance", International Journal of Artificial Intelligence and Applications (IJAIA), Vol. 9, No. 2, pp1-20.

[10] Winters, P., Adae, I., Silipo, R. (2014) "Anomaly Detection in Predictive Maintenance Anomaly Detection with Time Series Analysis", KNIME white paper.

[11] Winters, P., Silipo, R. (2015) “Anomaly Detection in Predictive Maintenance Time Alignment and Visualization", KNIME white paper. 
International Journal of Artificial Intelligence and Applications (IJAIA), Vol.9, No.3, May 2018

[12] Kotu, V., Deshpande, B. (2015) "Predictive Analytics and Data Mining”, Elsevier book.

[13] Wimmer, H., Powell, L. M. (2015) "A Comparison of Open Source Tools for Data Science", Proceedings of the Conference on Information Systems Applied Research. Wilmington, North Carolina USA.

[14] Al-Khoder, A., Harmouch, H., "Evaluating Four Of The most Popular Open Source and Free Data Mining Tools", International Journal of Academic Scientific Research, Vol. 3, No. 1, pp13-23.

[15] "Generic Web Service Client Node." 2018. [Online]. Available: https://www.knime.com/webservice-client

[16] A. Galiano, A. Massaro, D. Barbuzzi, L. Pellicani, G. Birardi, B. Boussahel, F. De Carlo, V. Calati, G. Lofano, L. Maffei, M. Solazzo, V. Custodero, G. Frulli, E. Frulli, F. Mancini, L. D’Alessandro, F. Crudele, (2016) "Machine to Machine (M2M) Open Data System for Business Intelligence in Products Massive Distribution oriented on Big Data", International Journal of Computer Science and Information Technologies, Vol. 7, No. 3, pp. 1332-1336, 2016.

[17] Grzegorz, J., Bartosz, A., (2015) “The Use Of IT Tools For The Simulation of Economic Processes", Information Systems in Management, Vol. 4, No. 2, pp 87-98.

[18] Dongare A.D., Kharde, R.R., Kachare, A.D. (2012) "Introduction to Artificial Neural Network", International Journal of Engineering and Innovative Technology, Vol. 2, No. 1, pp189-194.

[19] El-Khamisy, N., Ahmed Shawky Morsi El-Bhrawy, M. (2016) "Artificial Neural Networks in Data Mining”, Journal of Computer Engineering (IOSR-JCE), Vol. 18, No.6, pp. 55-59.

[20] Irfan, U., Fan, Y., Rehanullah, K., Ling, L., Haisheng, Y., Bing, G. (2017) "Predictive Maintenance of Power Substation Equipment by Infrared Thermography Using a Machine-Learning Approach", Energies, Vol. 10, No. 1987, pp1-13.

[21] Braun, H. (1993) “A Direct Adaptive Method for Faster Backpropagation Learning: the RPROP algorithm”, Proceedings of the IEEE International Conference on Neural Networks (ICNN), Vol. 16, pp586-591.

[22] Igel, C., Toussaint, M., Weishui, W. (2005) "RProp Using the Natural Gradient. International Series of Numerical Mathematics" Trends and Applications in Constructive Approximation. ISNM International Series of Numerical Mathematics, Vol. 151, pp1-15.

[23] Adhikari, N. C. D. (2018) "Prevention of Heart Problem Using Artificial Intelligence", International Journal of Artificial Intelligence and Applications (IJAIA), Vo., 9, No. 2, pp21-35.

[24] Fouad, R. H., ukattash, A. (2010) "Statistical Process Control Tools: A Practical guide for Jordanian Industrial Organizations", Jordan Journal of Mechanical and Industrial Engineering, Vol. 4, No. 6, pp693-700.

[25] Jawandhiya, P., "Hardware Design for Machine Learning", International Journal of Artificial Intelligence and Applications (IJAIA), Vol. 9, No. 1, pp63-84.

\section{Corresponding Author}

Alessandro Massaro: Research \& Development Chief of Dyrecta Lab s.r.l.

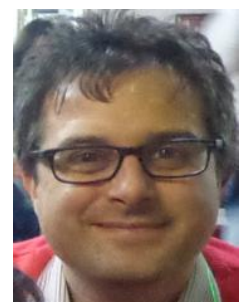

